Revista Científica General José María Córdova, Bogotá, Colombia, julio-diciembre, 2016

Ciencia y tecnología - Vol. 14, Núm. 18, pp. 215-243

ISSN 1900-6586

Cómo citar este artículo: Mejía, E. \& Ceballos, O. (2016, julio-diciembre). Medición contable de la sustentabilidad organizacional desde la Teoría Tridimensional de la Contabilidad. Rev. Cient. Gen. José María Córdova 14(18), 215-243

\title{
Medición contable de la sustentabilidad organizacional desde la Teoría Tridimensional de la Contabilidad"
}

Recibido: 4 de mayo de 2016 Aceptado: 12 de junio de 2016

Accounting Measurement for the Organizational Sustainability from the Three-dimensional Accounting Theory

Mesure comptable de la soutenabilité organisationnelle d’après la théorie tridimensionnelle de la comptabilité

Mensuração contábil da sustentabilidade organizacional a partir da Teoria Tridimensional da Contabilidade

\author{
Eutimio Mejía Soto ${ }^{a}$ \\ Olga Inés Ceballos Rincón ${ }^{b}$
}

* Informe del trabajo de investigación "Medición contable de la sustentabilidad organizacional. [Fase 2]. Un enfoque desde la Teoría Tridimensional de la Contabilidad T3C” realizado por el Grupo de Investigación en Contaduría Internacional Comparada, de la Universidad del Quindío, Armenia, Colombia. Trabajo adscrito al megaproyecto "Teoría Tridimensional de la Contabilidad T3C" (2014-2017), liderado por la Red de Investigación en Ciencias Económicas Administrativas y Contables (Rediceac).

a Universidad del Quindío, Armenia, Colombia. Contador Público de la Universidad del Quindío, Armenia, Colombia. Magíster y Doctorando en Desarrollo Sostenible de la Universidad de Manizales, Colombia. Docente integrante del Grupo de Investigación en Contaduría Internacional Comparada de la Universidad del Quindío, Armenia, Colombia. Email: eutimiomejia@uniquindio.edu.co

b Universidad del Quindío, Armenia, Colombia. Licenciada en Matemática y Computación, Especialista en Biomatemática de la Universidad del Quindío, Armenia, Colombia. Magíster en Educación Docencia de la Universidad de Manizales, Manizales, Colombia. Docente e Integrante del Grupo de Investigación de la Función Financiera de la Universidad del Quindío, Armenia, Colombia. Email: oiceballos@uniquindio.edu.co 
Resumen. La Teoría Tridimensional de la Contabilidad (T3C) integra la información de las dimensiones ambiental, social y económica de la riqueza que controla la organización, con el objeto de contribuir a la sustentabilidad de la misma. La protección y cuidado de ésta requiere de su medición y sus resultados permitirán evaluar la gestión en el control de los recursos administrados. La T3C supera al reduccionismo de la contabilidad económico-financiera. En tal sentido, reconstruye los fundamentos, conceptos y procedimientos de la teoría y la técnica contable, con el propósito de desarrollar un marco teórico-contable que incluya la riqueza ambiental y social, con el fin de contribuir a su conservación dinámica. Va más allá de los propósitos de explotación, con fines de rentabilidad económica, que ha caracterizado a la contabilidad tradicional. La medición de la sustentabilidad de la riqueza se calcula a través de fases estáticas, dinámicas y eco-sistémicas-interrelacionadas. La primera, analiza los cambios que se presentan en el activo o pasivo, en dos tiempos específicos: $\mathrm{t}, \mathrm{t} 1$. La segunda, estudia los movimientos de ingresos y egresos en un período de tiempo; la última, integra los enfoques de biocontabilidad, sociocontabilidad y contabilidad económica. El presente documento desarrolla la metodología de medición de los cambios de la riqueza en la organización en la fase estática: activos.

Palabras clave: activos, biocontabilidad, medición, riqueza, sociocontabilidad, sustentabilidad.

Abstract. The Three-dimensional Accounting Theory (3-DAT) comprises information from environmental, social and economic dimensions of wealth that takes control of the organization, in order to contribute to its sustainability. The protection and care of it requires its measurement, and the results will permit the evaluation of the management in the control of the managed resources. The 3-DAT overcomes the reductionism of the economic and financial accounting. In this sense, it reconstructs the foundations, concepts and procedures of the accounting theory and technique, with the purpose of developing a theoretical-accounting framework that includes the environmental and social wealth, with the aim of contributing to its dynamic conservation. It goes beyond the exploitation purposes, with economic profitability purposes, which are characteristic of the traditional accounting. The sustainability of wealth can be measured through interrelated static, dynamic and eco-systemic phases. The first one analyzes the changes that occur in the active or liability, in two specific times: $t, t 1$. The second one studies the movements of income and expenditure over a period of time; the last one incorporates approaches of biodiversity accounting, social accounting and economic accounting. This paper develops the measuring methodology for the wealth changes of the organization in the static phase, i.e., the active.

Keywords: assets, biodiversity accounting, measurement, social accounting, sustainability, wealth.

Résumé. La théorie tridimensionnelle de la comptabilité (TTC) intègre des informations sur les dimensions environnementales, sociales et économiques de la richesse contrôlée par l'organisation, en vue de contribuer à sa soutenabilité. La protection et le soin eux-mêmes implique sa mesure; et ses résultats permettront d'évaluer la gestion dans le contrôle des ressources gérées. La TTC dépasse le réductionnisme de la comptabilité économique et financière. En ce sens, elle établit les fondements, les concepts et les procédures de la théorie et de la technique de la comptabilité, en vue d'élaborer un cadre théorique comptable qui comprend la richesse environnementale et sociale, afin de contribuer à sa conservation dynamique. Elle demeure au-delà des fins d'exploitation, et de la rentabilité économique qui a caractérisé la comptabilité traditionnelle. On peut calculer la mesure de la soutenabilité de la richesse d'après des phases statiques, dynamiques et éco-systémiques. La première phase analyse les changements qui se produisent à l'actif ou le passif, en deux temps spécifiques: $t, t 1$. Deuxièmement, étudie les mouvements des produits et charges sur une période de temps; les dernières approches intégrées biocontabilidad, sociocontabilidad et la comptabilité économique. Cet article développe la méthodologie pour mesurer les changements dans l'organisation de richesse dans la phase statique: l'actif.

Mots-clés: actif, biocomptabilité, comptabilité sociale, mesure, richesse, viabilité. 
Resumo. A Teoria Tridimensional de Contabilidade (T3C) integra informaçóes de dimensóes ambientais, sociais e econômicas da riqueza controlada pela organização, a fim de contribuir para a sustentabilidade da mesma. Proteção e cuidado que exige medir e avaliar os seus resultados permitirão a gestão para controlar os recursos gerenciados. O T3C supera o reducionismo da contabilidade econômica e financeira. Neste sentido, ela recria os fundamentos, conceitos e procedimentos da teoria e da técnica da contabilidade, a fim de desenvolver uma base teórica contábil que inclui a riqueza ambiental e social, a fim de contribuir para a sua conservação dinâmica. A teoria vai além de fins de exploração para a rentabilidade econômica que tem caracterizado a contabilidade tradicional. A medição da sustentabilidade da riqueza é calculada por fases estáticas, dinâmicas e eco-sistêmicas inter-relacionadas. A primeira, analisa as mudanças que ocorrem no ativo ou passivo, em dois momentos específicos: $t, t 1$. Em segundo lugar, estuda os movimentos de receitas e despesas durante um período de tempo; as últimas, abordagens integradas bio-contabilidade, sócio-contabilidade e contabilidade econômica. Este trabalho desenvolve a metodologia para medir as mudanças na riqueza da organização na fase estática: ativo.

Palavras-chave: ativo, bio-contabilidade, medida, riqueza, sócio-contabilidade, sustentabilidade.

\section{Introducción}

La propuesta de medición contable de la sustentabilidad organizacional, desde la Teoría Tridimensional de la Contabilidad (T3C), se fundamenta en los trabajos que sobre el tema ha desarrollado la Red de Investigación en Ciencias Económicas y Administrativas (Rediceac) y el Grupo de Investigación en Contaduría Internacional Comparada; los cuales han permitido construir los cimientos para una nueva fundamentación epistemológica, metodológica, axiológica y teleológica de la contabilidad. Las mediciones expuestas se describirán en el marco teórico y se sustentarán en criterios tanto conceptuales como teóricos.

El presente documento expone el origen y desarrollo de la T3C a través de sus publicaciones; como una propedéutica para comprender y medir la sustentabilidad de la riqueza que controla la organización. Se desarrolla el método de existencia fase activos, objeto de este escrito, en la cual se analizará el cambio de los mismos en el tiempo. El estudio y comprensión de la sustentabilidad requiere de la lectura integral de la existencia, circulación e impactos en las riquezas en las tres dimensiones, calculados a través de los modelos: biocontabilidad, socio-contabilidad y contabilidad económica, en las fases seńaladas. Cada fase ofrece una panorámica de aproximación a la sustentabilidad de la riqueza, el conjunto de fases permite comprender integralmente la situación y evolución de la misma.

El texto desarrolla los siguientes apartados, un marco teórico en el cual se exponen los aspectos esenciales de la T3C, entre ellos, la definición de contabilidad, biocontabilidad, sociocontabilidad y contabilidad económica, al igual que las proposiciones básicas que sustentan la teoría. El marco histórico relata cronológicamente la evolución de la T3C a través de las publicaciones que paulatina y sistemáticamente van construyendo la teoría conceptual y técnica de una propuesta dinámica-integradora de la contabilidad. Las definiciones básicas asociadas al método de existencia fase activos son presentadas para contribuir a una mejor comprensión de las fórmulas presentadas. 
El cuerpo del trabajo se desarrolla bajo el título "Medición de la sustentabilidad de la riqueza en la organización”, se exponen las mediciones de cada dimensión de la riqueza y el cálculo integral en cada proceso y en la organización. Los resultados de la medición de la sustentabilidad en los procesos, en los tipos de riqueza y la tridimensional de la organización, permiten identificar, en términos espacio-temporales, los impactos positivos y negativos que las acciones-omisiones de la organización generan frente a la riqueza.

La información obtenida permite calificar las organizaciones conforme a 27 escańos que combinan la calificación de sustentable, insustentable y estable, para las dimensiones de la riqueza ambiental, social y económica. La evaluación de la gestión organizacional frente a la riqueza permitirá la rendición social de cuentas y la toma de decisiones con una información integral de los efectos que reflejan el actuar de la organización en función de la anhelada sustentabilidad.

\section{Marco teórico}

Para poder realizar una medición adecuada de la T3C, es importante conocer teóricamente los diferentes conceptos que la integran.

- La contabilidad es la ciencia social aplicada [tecnociencia] que estudia la valoración cualitativa y cuantitativa de la existencia y circulación de la riqueza ambiental, social y económica controlada por la organización, utilizando diversos métodos que le permiten cumplir su función de evaluar la gestión que la organización ejerce sobre la riqueza mencionada, con el fin de contribuir a la óptima acumulación, generación, distribución y sustentabilidad de la misma. ${ }^{1}$ (Mejía et al., 2015, 29).

- La biocontabilidad es la disciplina social que estudia la valoración cualitativa y cuantitativa de la existencia y circulación de la riqueza ambiental controlada por la organización, utilizando diversos métodos que le permiten cumplir su función de evaluar la gestión que la organización ejerce sobre la riqueza ambiental, con el fin de contribuir a la óptima acumulación, generación, distribución y sustentabilidad de la mencionada riqueza (ambiental) (Mejía et al., 2015, 52).

- La sociocontabilidad es la disciplina social que estudia la valoración cualitativa y cuantitativa de la existencia y circulación de la riqueza social controlada por la organización, utilizando diversos métodos que le permiten cumplir su función de evaluar la gestión que la organización ejerce sobre la riqueza social, con el fin de contribuir a la óptima acumulación, generación, distribución y sustentabilidad de la mencionada riqueza [social]. (Mejía et al., 2015, 53).

- La contabilidad económica es la disciplina social que estudia la valoración cualitativa y cuantitativa de la existencia y circulación de la riqueza económica controlada por la organización, utilizando diversos métodos que le permiten cumplir su función de evaluar

1 Diversos autores han definido la contabilidad desde otras concepciones, alcances y aplicaciones (Fowler, 2007, 9; García Benau, 2004, 29; García Casella, 2001, 13; García García, 1997, 133; Lopes de Sa, 1995, 14-15; Mattesich, 2002, 52). 
la gestión que la organización ejerce sobre la riqueza económica, con el fin de contribuir a la óptima acumulación, generación, distribución y sustentabilidad de la mencionada riqueza [económica]. (Mejía et al., 2015, 29).

Por otro lado, para una mejor comprensión del tema, se elaboró la tabla 1.

Tabla 1. Modelos de la Teoría Tridimensional de la Contabilidad

\begin{tabular}{llll}
\hline \multicolumn{1}{c}{ Criterio a evaluar } & \multicolumn{1}{c}{ Biocontabilidad } & \multicolumn{1}{c}{ Sociontabilidad } & \multicolumn{1}{c}{ Contabilidad económica } \\
\hline Objeto de estudio & $\begin{array}{l}\text { Valoración ambiental de la } \\
\text { riqueza ambiental controla- } \\
\text { da por la organización. }\end{array}$ & $\begin{array}{l}\text { Valoración social de la rique- } \\
\text { za social controlada por la } \\
\text { organización. }\end{array}$ & $\begin{array}{l}\text { Valoración económica de la } \\
\text { riqueza controlada por la or- } \\
\text { ganización. }\end{array}$ \\
\hline Finalidad & $\begin{array}{l}\text { La sustentabilidad de la rique- } \\
\text { za ambiental. }\end{array}$ & $\begin{array}{l}\text { La sustentabilidad de la ri- } \\
\text { queza social. }\end{array}$ & $\begin{array}{l}\text { Mantenimiento del capital } \\
\text { económico. }\end{array}$ \\
\hline $\begin{array}{l}\text { Criterios } \\
\text { de reconocimiento }\end{array}$ & $\begin{array}{l}\text { La existencia de un recurso } \\
\text { natural. }\end{array}$ & $\begin{array}{l}\text { La existencia de un recurso } \\
\text { útil en su función social. }\end{array}$ & $\begin{array}{l}\text { Beneficio o sacrificio econó- } \\
\text { mico de la partida y medi- } \\
\text { ción monetaria confiable }\end{array}$ \\
\hline $\begin{array}{l}\text { Presentación } \\
\text { de información }\end{array}$ & Estados contables ambientales & Estados contables sociales & $\begin{array}{l}\text { Estados contables económicos } \\
\text { (entre ellos los estados finan- } \\
\text { cieros) }\end{array}$ \\
\hline Beneficios perseguidos & Sustentabilidad ambiental & Beneficios Sociales & Beneficios Económicos \\
\hline $\begin{array}{l}\text { Unidad de medida } \\
\text { Unidades de valor ambiental } \\
\text { (UVA) }\end{array}$ & $\begin{array}{l}\text { Unidades de valor social } \\
\text { (UVS) }\end{array}$ & Unidades monetarias (UM) \\
\hline $\begin{array}{l}\text { Método } \\
\text { de presentación }\end{array}$ & Múltiples métodos & Múltiples métodos & Partida doble \\
\hline Estados contables tridimensionales (integrados) & & \\
\hline $\begin{array}{l}\text { Evaluación de la sustentabilidad (integrada) de la organización. } \\
\text { Integración de diferentes unidades de medida/valoración }\end{array}$ & \\
\hline
\end{tabular}

Fuente: elaboración propia

La tabla 1 muestra las diferencias conceptuales y prácticas que subyacen en los modelos que constituyen la tridimensionalidad contable. La realidad objeto de estudio forma parte del primer factor de distinción. En contraste, la biocontabilidad estudia la valoración ambiental; la sociocontabilidad, la valoración social; y la contabilidad económica, la valoración económica. La contabilidad tridimensional las integra, comprendiendo su interrelación e inter-dependencia integral. El fin perseguido en cada modelo es la sustentabilidad-mantenimiento de su dimensión de la riqueza, factor que deberá conciliarse en la evaluación integral de la sustentabilidad. Los criterios de reconocimiento evidencian que cada campo de la riqueza se expresa de forma diferente, requiriendo criterios específicos para su inclusión, donde los aspectos tenidos en cuenta en una dimensión no son pertinentes para ser aplicable en las otras. 
Las particularidades de cada una de las dimensiones de la riqueza requieren que se presenten estados-informes contables en los tres campos, los estados financieros tradicionales no son los adecuados para representar la realidad ambiental y social. Cada modelo evidencia el beneficio que el hombre pretende lograr con la información elaborada, evidenciándose las diferencias entre las concepciones antropocéntricas y biocéntricas. Las dimensiones ambiental y social de la riqueza deben ser representadas en las unidades de valor ambiental y unidades de valores social, propuestas bajo la T3C.

La partida doble se considera el método más común de representación de la riqueza económica, no siendo el único. La biocontabilidad y la sociocontabilidad deberán indagar nuevos métodos de representación. Los estados tridimensionales constituyen el reconocimiento a la integralidad e indivisibilidad de la realidad, la conexión y articulación de las dimensiones, la condición sistémica de unidad de la vida, en las multi-relaciones y la convivencia dinámica de pluralidad de causas-efectos, en tiempos y espacios indeterminables.

Desde el punto de vista teórico de la Teoría Tridimensional de la Contabilidad, es importante resaltar que ésta se sustenta en las siguientes proposiciones:

- La contabilidad es una ciencia social aplicada que desarrolla componentes tecnológicos y técnicos. Tiene un campo positivo empírico e inductivo y otro campo normativo deductivo y subjetivo, con criterios estratégicos y éticos.

- El objeto material y formal de estudio de la contabilidad incluye la riqueza ambiental, social y económica.

- La contabilidad se complementa con diferentes ramas del saber, no implica relaciones de dominio o dependencia. Los problemas de la sociedad y la solución de los mismos requieren la participación activa de todos los saberes científicos y no científicos.

- La contabilidad permite rendir cuentas (pasado), mostrar el presente, predecir y prescribir el futuro. Cumplir estos objetivos se logra con el avance en cuatro etapas, la descripción, la explicación, la predicción y la prescripción de la valoración cualitativa y cuantitativa de la existencia y circulación de la riqueza ambiental, social y económica controlada por la organización.

- La finalidad de la contabilidad tridimensional se sustenta en una concepción ética biocéntrica, no se limita a dar cuenta de la realidad en términos de neutralidad y objetividad, además, y de manera preponderante, tiene una finalidad anclada en aspectos axiológicos sustentados en el deber ser de la sociedad.

- La contabilidad puede utilizar diversos métodos de representación tales como la partida simple, la partida doble, partidas múltiples, entre otros.

- La T3C propone el uso cualitativo y cuantitativo de unidades de medida, estas últimas monetarias y no monetarias, reconociendo que en cada dimensión se utilizarán criterios de medición y valoración intrínseca y extrínseca diferenciales, dependiendo la realidad objeto de estudio y del modelo contable aplicado. La valoración intrínseca mide-valora un activo a partir de la existencia o afectación de un activo, independiente de los beneficios que represente para el hombre, tal como lo hace la valoración extrínseca. 
- La T3C aporta a la consolidación del saber científico contable y es un factor de conexión entre el campo teórico y aplicado de la contabilidad, esta última, fundamentada en la responsabilidad ambiental, social y económica que articula todos los saberes, las profesiones y oficios y, ante todo, los hombres en su condición de sujetos moralmente enjuiciables por sus acciones y omisiones en la construcción de un mundo mejor. La responsabilidad del profesional contable debe evaluarse por su contribución a una sociedad más justa, equitativa, solidaria y respetuosa de todas las formas y manifestaciones de vida, su compromiso no es con la organización sino con la sociedad presente y las generaciones futuras.

La medición contable de la sustentabilidad organizacional desde la T3C constituye un nexo entre la fundamentación teórico-conceptual y la implementación de técnicas cualitativas y cuantitativas para su medición-valoración (Schuschny y Soto, 2009, 81-92); proceso que se desarrolla en cuatro fases:

a. Medición de la sustentabilidad de la riqueza en la organización, método de existencia fase activos. Analiza los cambios presentados en un activo (x) en dos fechas de tiempo específicas $(\mathrm{t} ; \mathrm{t}-1)$.

b. Medición de la sustentabilidad de la riqueza en la organización, método de existencia fase pasivos. Analiza los cambios presentados en un pasivo (x) en dos fechas de tiempo específicas $(\mathrm{t}$; $\mathrm{t}-1)$.

c. Medición de la sustentabilidad de la riqueza en la organización, método circulación fase ingresos-egresos. Analiza los flujos presentados en un recurso (x) en un período de tiempo determinado.

d. Medición de la sustentabilidad de la riqueza en la organización, método correlacional, fase eco-socio-sistémica. Analiza la existencia y circulación de los recursos de manera holística, integral y sistémica.

\section{Marco histórico de Teoría Tridimensional de la Contabilidad}

La Teoría Tridimensional de la Contabilidad T3C es el resultado de un conjunto de investigaciones que fueron realizadas por los grupos de investigación y escuelas de investigación adscritos a la Red de Investigación de Ciencias Económicas y Administrativas (Rediceac). Las publicaciones previas relacionadas con la T3C fueron:

a. Mora, Montes y Mejía (2005) en Efectos de la regulación contable internacional proponen que las acciones de las organizaciones tienen efectos económicos, sociales y ambientales; se afirma que la contabilidad tradicional sólo reconoce parte de los impactos económicos, desconociendo los impactos en las otras dos dimensiones en las que la organización debe informar. Cuando las organizaciones presentan solamente información económica, dicha situación es incompleta y sesgada, la organización debe 
presentar información integral de sus actividades e impactos en todos los niveles de la organización y la sociedad.

b. Montes, Mejía y Montilla $(2005,210)$ en el texto Contabilidad emergente y Mejía, Montes y Botero $(2006,113)$ proponen para la contabilidad el Programa de investigación científico PIC "ético-socio-humanístico", se afirma que la contabilidad debe abandonar el carácter eminentemente económico para crear un nuevo núcleo, ubicando la relación naturaleza-hombre-economía como estructura básica. Utilizando el lenguaje de Imre Lakatos, señalan que el PIC económico en la contabilidad es un programa regresivo, agotado e insuficiente para dar respuesta a las exigencias sociales, en tal sentido, se sustenta que la contabilidad ambiental y la contabilidad social constituyen opciones de PIC contables de carácter progresivo, siendo estas temáticas campos de investigación poco explorados por la disciplina.

c. Montes, Mejía y Valencia (2006, 98 y 99) en el texto paradigmas en contabilidad sintetizan la propuesta de un nuevo modelo de contabilidad, orientado "al mejoramiento de las condiciones de vida de las comunidades, contribuir a la protección del medio ambiente y garantizar que las generaciones puedan satisfacer sus necesidades como lo prescribe el concepto de desarrollo sostenible ${ }^{2}$.

d. Mejía (2010) en el texto Contabilidad ambiental, sustenta que el modelo contable financiero [nacional -Col-PCGA e internacional- IAS-IFRS] no es pertinente para dar cuenta de la realidad ambiental, señala, además, que es diferente a la contabilidad ambiental $^{3}$, la contabilidad de gestión ambiental y la contabilidad financiera ambiental, indicando que la primera requiere su formulación conceptual y su desarrollo técnico.

e. Mejía, Montes y Montilla (2010) en el artículo "Análisis de los métodos de medición de las cuentas ambientales en el modelo contable financiero y concepciones alternativas", analizan más de treinta métodos de valoración de los recursos ambientales, se concluye que las valoraciones se basan en aspectos fundamentalmente económicos; en tal sentido, se advierte la necesidad de diseñar o proponer métodos de medición de los recursos ambientales sustentados en criterios no económicos, donde se reconozca el valor de la naturaleza, no por el beneficios que representa para el hombre en términos de utilidad mercantil únicamente, sino que también se reconozcan sus beneficios ambientales y sociales, los cuales no siempre es posible representar en términos monetarios. Existen recursos naturales para los cuales su valor ambiental y social es tan alto, que no existe representación monetaria que pueda representar el verdadero valor de dicho recurso.

f. Mejía (2011) en el artículo "Análisis de los criterios de reconocimiento de las cuentas ambientales en los estándares internacionales de contabilidad y reportes financieros IAS-IFRS”, señala que, tradicionalmente, la contabilidad ha utilizado a los valores de mercado como criterios de reconocimiento, es decir, solo se incluyen en los estados contables las partidas que representan beneficios económicos y son susceptibles de me-

2 El término "desarrollo sostenible" posteriormente fue reemplazado por la expresión sustentabilidad.

3 El término biocontabilidad es acuñado desde la Teoría Tridimensional de la Contabilidad T3C para distinguir el enfoque financiero y el enfoque físico-sustentable de la relación contabilidad y naturaleza. 
dición monetaria. Se propone que la contabilidad ambiental debe tener unos criterios de reconocimiento a partir de la existencia natural del recurso, independiente de los beneficios económicos que pueda representar para el hombre; por lo tanto, se sugiere una medición cualitativa y cuantitativa no monetaria y monetaria no tradicional.

g. Mejía y Montes (2011 y 2011a) en la ponencia "Estructura general de la teoría pura, los modelos y sistemas en contabilidad" y en el artículo "Fundamentación teórica de los modelos en contabilidad" presentan una nueva definición general de contabilidad como

la ciencia social aplicada que estudia la valoración cualitativa y cuantitativa de la existencia y circulación de las riquezas (ambiental, social y económica) controladas por las organizaciones, utilizando diversos métodos que le permiten evaluar el control múltiple [gestión] que la organización ejerce sobre la mencionada riqueza, con el fin de contribuir a la acumulación, generación, distribución y sostenibilidad integral de la misma. Mejía y Montes (2011a).

h. Mejía y Montes (2012) en el artículo "Análisis de los conceptos de capital, mantenimiento de este y su relación con la riqueza ambiental" establecen que la contabilidad tiene tres modelos contables: ambiental, social y económico; que corresponden a igual número de dimensiones de la contabilidad y tipos de riqueza que la organización debe generar, acumular y sostener de manera integral.

i. Mejía, Montes y Arango (2012) en el artículo "Aproximación al sistema de contabilidad ambiental y económica integrada SCAEI de la ONU”, resaltan que los desarrollos de la macro-contabilidad ambiental pueden aportar significativamente para el avance de la contabilidad micro-ambiental, teniendo presente la necesidad de sustentar la valoración de la riqueza ambiental a partir de criterios intrínsecos que permitan su protección y cuidado.

j. Mejía y Vargas (2012) en el artículo "Contabilidad para la sostenibilidad ambiental y social" seńalan que, tradicionalmente, la contabilidad ha sido concebida como una disciplina de carácter económico, limitada a la protección del capital financiero de los inversionistas y como factor determinante para el cálculo de las imposiciones fiscales. La nueva contabilidad, se desarrolla como una ciencia social que evalúa la gestión de la organización en la generación y sostenibilidad de los diferentes tipos de riqueza que controla; desde esta concepción, se establece una escala de prioridades en la protección del patrimonio de las entidades, donde los intereses económicos están supeditados a los propósitos superiores de protección y de mantenimiento [dinámico] de la riqueza ambiental y social. La dimensión contable ambiental debe ser incluyente y fundada en una ética de la vida. El respeto por la naturaleza no puede estar sustentado en los beneficios que ésta representa para el hombre, el valor de la naturaleza descansa en sí mismo, en su propia condición, en su existencia, no en función de su utilidad para los fines del hombre. Cuánta humildad requiere tener el ser humano para comprender que es una especie más de la naturaleza y no la especie superior de la misma.

k. Mejía, Mora y Montes (2013) en el artículo "Adscripción de la contabilidad en la estructura general del conocimiento" se presenta la Teoría tridimensional de la contabilidad como una nueva visión amplia, holística e integral de la contabilidad, afirmando 
que "la ciencia contable debe dar cuenta de la dimensión ambiental, la social y la económica, pero el componente económico no es el más importante”.

1. Mejía et al. (2014) en "Teoría tridimensional de la contabilidad T3C (versión 2.0): desarrollos, avances y temas propuestos" " corresponde a una revisión de la publicación "Estructura conceptual de la teoría tridimensional de la contabilidad"5 (2013), en conjunto, constituyen la presentación de la estructura de la contabilidad tridimensional expuesta en cinco componentes, filosófico, contextual, tecno-conceptual, tecno-procedimental y de validación; la versión 2.0, además de un ajuste conceptual, propone temas de investigación requeridos para el desarrollo y consolidación de la teoría en referencia.

m. Mejía (2014) en "La teoría tridimensional de la contabilidad: apuntes de la economía ecológica (Martínez y Roca)" afirma que la contabilidad es una ciencia social aplica$\mathrm{da}$, con componentes tecnológicos y técnicos; su objeto de estudio incluye la riqueza ambiental, social y económica; se eleva a la categoría de imperativo categórico el mantenimiento [dinámico] del capital natural y se resalta la necesidad de superar la visión débil de la sostenibilidad para orientar el accionar hacia un modelo integral de sustentabilidad que reconozca el carácter holístico, integral y sistémico de la naturaleza. La contabilidad ecológica puede hacer aportes grandes y significativos al desarrollo de una contabilidad en función de la naturaleza.

n. Mejía (2014) en "Biocontabilidad: hacia una definición de una nueva disciplina contable", Mejía et al. (2014c) en "Marco conceptual de la biocontabilidad", Mejía y Quintero (2015) en "Biocontabilidad: un enfoque para la sustentabilidad" y Mejía, Montes y Mora (2015) en "Marco conceptual de la contabilidad ambiental" se construyen los primeros avances en uno de los componentes de la Teoría Tridimensional de la Contabilidad (T3C), como es la biocontabilidad; se identifican las diferencias con la contabilidad ambiental y se propone un marco conceptual específico para la contabilidad en su relación con la naturaleza.

o. Mejía $(2015,190)$ en "Aportes a la biocontabilidad desde la bioeconomía de GeorgescuRogen" concluyen que la economía es un subsistema abierto que tiene implicaciones en otros subsistemas que debe considerar con mayor responsabilidad, por ejemplo, desde la captura de recursos del ambiente hasta la generación y emisión de desechos. El desarrollo de la disciplina de biocontabilidad debe nutrirse de diferentes campos del saber, entre ellos la bio-economía. Las ciencias tecnológicas y técnicas deben estar al servicio de la naturaleza, de la vida y, por ende, del hombre, como una especie más dentro de todas las manifestaciones de vida.

p. Mejía y Serna (2015) en "La contabilidad en función de la sustentabilidad: una mirada desde el desarrollo económico alternativo” señalan que

La contabilidad ha sido instrumentalizada conforme a la lógica de la financiarización, se encuentra al servicio del proceso de acumulación de riqueza económica a cualquier

\footnotetext{
4 Versión ampliada en el libro "Teoría Tridimensional de la Contabilidad. Versión 2.0" (Mejía et al., 2015).

5 Versión ampliada en el libro "Teoría Tridimensional de la Contabilidad”. (Mejía et al., 2014a).
} 
costo ambiental y social. Se propone una ruptura paradigmática de la contabilidad, un repensar de su orientación ética, de forma que asuma su estatus de cientificidad, su función y finalidad de protectora del interés general, una vez que la sociedad históricamente le ha encomendado la misión de ser guardián de la riqueza para el bien común, en beneficio de la presente y las futuras generaciones en todas las formas y expresiones de vida. (p. 117 y 118).

\section{Metodología}

La investigación "Medición contable de la sustentabilidad organizacional [fase estática-activos]", se desarrolló utilizando el método inductivo, a través de un enfoque cualitativo y cuantitativo que propone un modelo de medición de la sustentabilidad de los activos y la riqueza, desde lo unidimensional a lo tridimensional; el cálculo tiene en cuenta el estado de los activos en dos puntos específicos en el tiempo $\mathrm{t}, \mathrm{t}-1$.

El carácter deductivo del modelo de medición propuesto proviene de la concepción moral-social de la ciencia contable, que considera su contribución a la sustentabilidad de la riqueza en sus dimensiones ambiental, social y económica. El trabajo presenta un carácter descriptivo, explicativo y propositivo que sustenta la evaluación de la gestión organizacional por medio de la medición contable de estado de los activos y la riqueza en cada uno de los procesos.

Descriptivo, porque da cuenta del estado de los activos-riqueza en unos momentos específicos en el tiempo. Explicativo, porque con la ayuda de la fase dinámica puede identificar las causas que dieron origen a los cambios de los mismos (activos). Propositivo, porque pretende fundamentar acciones de mejora en los procesos insustentables y fortalecer las acciones que contribuyen positivamente a la sustentabilidad.

Como se mencionó en la introducción, antes de realizar el proceso de formulación, se describirán las definiciones de los términos que serán utilizados.

\section{Definiciones básicas}

La propuesta de medición de la sustentabilidad de la riqueza controlada por la organización, se fundamenta en la T3C (versión 2.0), en congruencia las definiciones básicas.

- Activo: Los activos son el conjunto de bienes y derechos [tangibles o intangibles] de tipo natural, social o económico controlados por la organización ${ }^{6}$, ya sea a través del dominio, propiedad, control, uso, posibilidad de disposición y/o explotación directa o indirecta del mismo; del cual la organización tiene la posibilidad de obtener algún tipo de beneficio ya sea ambiental, social o económico (Mejía et al., 2015, 39; Mejía et al., 2014a, 23).

6 Con respecto a la protección de los recursos y los mecanismos para lograrlo ver Ostrom (2011) y lectura complementaria North (2012). 
- Pasivos: corresponden al conjunto de obligaciones ${ }^{7}$ que tiene la organización, las cuales deberán ser cubiertas en el presente o futuro, surgidas como resultado de actos y hechos pasados en los cuales la organización, de forma directa o indirecta, participa por acción u omisión, de forma voluntaria o involuntaria, en el uso, consumo, disposición y/o explotación de algún tipo de riqueza, que era directa o indirectamente controlada por otra(s) organización(es) o que estando bajo propio control, tenía el deber de conservarla y no lo hizo. El cumplimiento de las obligaciones implica que la(s) organización(es) debe(n) transferir parte de su riqueza controlada a otra(s) organización(es) para dar cumplimiento con la obligación existente (Mejía et al., 2015, 39; Mejía et al., 2014a, 23-24).

- Ingreso: "Son los incrementos cualitativos y/o cuantitativos de los activos adquiridos o generados en un período determinado diferente de los movimientos patrimoniales, representados en la entrada de nuevos activos o el mejoramiento de la calidad de los existentes; tales entradas o aumentos de los activos son el resultado de las acciones y/u omisiones del hombre, o consecuencia de la dinámica autónoma de la propia riqueza y/o su interacción en el medio" (Mejía et al., 2015, 40) (ver también: IFRSF, 2014, 4.29).

\section{- Egresos:}

Son los decrementos cualitativos y/o cuantitativos de los activos en un período determinado, diferentes a los movimientos patrimoniales, representados en la salida de activos o el desmejoramiento de la calidad de los existentes; tales salidas o reducciones, son el resultado de las acciones y/u omisiones de la organización, o consecuencia de la dinámica autónoma de la propia riqueza y su interacción en el medio. El egreso de un activo individual o de un conjunto de ellos implica una disminución absoluta o parcial de la capacidad potencial o real de un activo o de un conjunto de ellos de cumplir las mismas funciones que antes de presentarse el decremento. (Mejía et al., 2015, 40-41), (IFRSF, 2014, 4.33; Cañibano, 2000, 87; Mejía, 2010, 49).

- Riqueza: el término riqueza contablemente se refiere a toda cosa que existe en la naturaleza o en la mente de los hombres, ya sea corporal o incorporal, mueble o inmueble, natural o artificial, concreta o abstracta, la cual pueda ser apropiada y/o controlada por el hombre, el cual podrá tener dominio, uso, goce, capacidad de modificación y/o disposición de la misma, ejerciendo algún impacto o alteración voluntario o involuntario, sea con propósitos de utilización directa o indirecta o por condiciones eventuales y/o fortuitas, que como consecuencia sufra algún tipo de transformación positiva o negativa $^{8}$ [ver conceptos de capital en Biondi, 2008; IIRC, 2013].

- Sustentabilidad de la riqueza: proceso que, de forma continua, autónoma o por intervención de la naturaleza o de la acción humana permite la perdurabilidad, conservación,

7 Relación que existe entre dos personas naturales o jurídicas, en la cual una de ellas se compromete para con la otra o ambas se comprometen para con el cumplimiento de una obligación de dar, hacer o no hacer (concepto jurídico). La T3C considera que la responsabilidad organizacional supera los límites del derecho positivo, para anclarse en el marco de una justicia ambiental, con alcance intra e intergeneracional.

${ }^{8}$ La definición de riqueza debe ser entendida en congruencia con el objeto material y forma de la contabilidad, con su función y su finalidad. 
mantenimiento dinámico ${ }^{9}$ y/o crecimiento de la riqueza controlada por la organización en una o todas sus dimensiones [ambiental, social y económica]. La sustentabilidad de la riqueza es uno de los fines básicos o genéricos de la contabilidad, compartido con otros campos del saber; se representa en términos de calidad y cantidad, en función de los beneficios reales o potenciales que represente para la vida en general y/o para el hombre en particular. La contribución organizacional a la sostenibilidad corresponde a su responsabilidad intra e inter generacional, a través de procesos espacio-temporales adecuados de acumulación, generación, distribución y circulación endo y exo-organizacional (Mejía et al., 2015, 45); (para otras definiciones ver también: Caro, 2011, 35; Boada, Rocchi y Kuhndt, 2015, 19; Labandeira, León y Vázquez, 2006, 28): (para Desarrollo Sostenible, ver Brundtland, 1987, 55).

\section{Medición de la sustentabilidad de la riqueza en la organización}

Las organizaciones desarrollan procesos [conjunto de actividades] que impactan las dimensiones de la riqueza ambiental, social y/o económica; donde el movimiento de un activo afecta la sustentabilidad positiva o negativa de otros, es decir, los activos y las dimensiones están correlacionados indefectiblemente. La contabilidad debe incluir en su universo discursivo las mediciones en los tres campos en referencia, para ello debe desarrollar metodologías que den cuenta de la sustentabilidad por procesos, dimensiones e interrelaciones [integralidad]. A continuación, se desarrolla el modelo de medición de la sustentabilidad de la riqueza en la organización fase estática-activos:

\section{Medición unidimensional de la riqueza}

a. Medición de los activos de la dimensión ambiental [Ada]: se calcula a través de la suma de los activos (x) en la dimensión ambiental de la riqueza, multiplicada por su factor de conversión de unidades, medido en un tiempo determinado.

$$
A d a=\sum_{x=1}^{n} A a_{x} p_{x}
$$

Donde

$x=$ Cada activo de la dimensión ambiental de la riqueza $(x=1, \ldots, n)$

$A a_{x}=$ Activo $(x)$ de la dimensión ambiental de la riqueza.

$P_{x}=$ factor unidimensional de conversión de unidades de cada activo $(x)$ de la dimensión ambiental de la riqueza.

9 Sostenibilidad no es lo mismo que inmovilidad... hasta los sistemas vírgenes están en permanente variación, lo que involucra la renovación y destrucción de sus componentes, adaptándose a los cambios de sus ambientes, co-evolucionando junto con ellos (Holling, 1973, citado por Gallopin, 2003, 21). 
b. Medición de los activos de la dimensión social [Ads]: se calcula a través de la suma de los activos (y) en la dimensión social de la riqueza, multiplicada por su factor de conversión de unidades, medido en un tiempo determinado.

$$
A d s=\sum_{y=1}^{k} A s_{y} p_{y}
$$

Donde

$y \quad=$ Cada activo de la dimensión social de la riqueza $(y=1 \ldots, k)$

$A s_{y}=$ Activo $(y)$ de la dimensión social de la riqueza.

$P y=$ factor unidimensional de conversión de unidades de cada activo (y) de la dimensión social de la riqueza.

c. Medición de los activos de la dimensión económica [Ade]: se calcula a través de la suma de los activos ( $\mathrm{z}$ ) en la dimensión económica de la riqueza, multiplicada por su factor de conversión de unidades, medido en un tiempo determinado.

$$
A d e=\sum_{Z=1}^{q} A e_{z} P_{Z}
$$

Donde

$z \quad=$ Cada activo de la dimensión social de la riqueza $(z=1 \ldots, q)$

$A e_{z}=$ activo $(y)$ de la dimensión social de la riqueza.

$P_{z}=$ factor unidimensional de conversión de unidades de cada activo (z) de la dimensión económica de la riqueza

\section{Medición de la sustentabilidad de los recursos que conforman la riqueza en cada dimensión}

a. Sustentabilidad de un activo ambiental $\left(\mathrm{SAa}_{\mathrm{x} \Delta}\right)$ : se calcula a través de la diferencia de la medición de un activo ambiental $(\mathrm{x})$ en un período de tiempo $(t-1$ a $t)$

Donde

$$
S A a_{x \Delta}=A a_{x t}-A a_{x t-1}
$$

Aa = Activo ambiental

$\mathrm{x} \quad=$ Cada activo de la riqueza en la dimensión ambiental $(x=1 \ldots, n)$

$\Delta \quad=$ Periodo de tiempo

$S A a_{x \Delta}=$ Sustentabilidad del activo ambiental ( $x$ ) en un periodo de tiempo $(t-1$ a $t)$ 
$A a_{x t}=$ Activo ambiental $(x)$ en el tiempo $(t)$

$A a_{x t-1}=$ Activo ambiental $(x)$ en el tiempo $(t-1)$

b. Sustentabilidad de un activo social $\left(\mathrm{SAs}_{\mathrm{y} \Delta}\right)$ : se calcula a través de la diferencia de la medición de un activo social (y) en un período de tiempo ( $\mathrm{t}-1 \mathrm{a} \mathrm{t})$

$$
S A s_{y \Delta}=A s_{y t}-A s_{y t-1}
$$

Donde

As = Activo social

$y=$ Cada activo de la riqueza en la dimensión social $(y=1 \ldots, k)$

$S A s_{y \Delta}=$ Sustentabilidad del activo social $(y)$ en un periodo de tiempo $(t-1$ a $t)$

As ${ }_{y t}=$ Activo social $(y)$ en el tiempo $(t)$

$A s_{y t-1}=$ Activo social $(y)$ en el tiempo $(t-1)$

c. Sustentabilidad de un activo económico $\left(\mathrm{SAe}_{\mathrm{z} \Delta}\right)$ : se calcula a través de la diferencia de la medición de un activo económico $(\mathrm{z})$ en un período de tiempo $(t-1$ a $t)$

$$
S A e_{z \Delta}=A e_{z t}-A e_{z t-1}
$$

Donde

Ae = Activo económico

$z \quad=$ Cada activo de la riqueza en la dimensión económica $(z=1 \ldots, q)$

$S A e_{z \Delta}=$ Sustentabilidad del activo económico (z) en un periodo de tiempo ( $t-1$ a $\left.t\right)$

$A e_{z t}=$ Activo económico ( $z$ ) en el tiempo ( $\left.t\right)$

$A z_{z t-1}=$ Activo económico $(z)$ en el tiempo $(t-1)$

\section{Medición de la sustentabilidad unidimensional de la riqueza en los procesos (fase activos)}

a. Medición de la Sustentabilidad Ambiental en un Proceso [Sap $\mathrm{S}_{\mathrm{i} \Delta}$ ]. Se calcula a través de la sumatoria de la sustentabilidad de cada activo de la riqueza ambiental en el proceso (i), multiplicado por su factor de conversión, medido en un período de tiempo determinado.

$$
\operatorname{Sap}_{i \Delta}=\sum_{x=1}^{n}\left(A a_{i x t}-A a_{i x t-1}\right) p_{x}
$$

Donde:

Aa = Activo ambiental

$x \quad=$ Cada activo de la riqueza en la dimensión ambiental $(x=1 \ldots, n)$ 
$i \quad=$ Cada proceso $(i=1 \ldots, m)$

$A a_{x t}=$ Activo ambiental $(x)$ en el tiempo ( $\left.t\right)$ en el proceso ( $\left.i\right)$

$A a_{x t-1}=$ Activo ambiental $(x)$ en el tiempo ( $\left.t-1\right)$ en el proceso ( $\left.i\right)$

$P_{x} \quad$ factor unidimensional de conversión de unidad de cada activo $(x)$ en la dimensión ambiental de la riqueza en los procesos

b. Medición de la Sustentabilidad Social en un Proceso [Ssp $\left.\mathrm{S}_{\mathrm{i} \Lambda}\right]$. Se calcula a través de la sumatoria de la sustentabilidad de cada activo de la riqueza social en el proceso (i), multiplicado por su factor de conversión, medido en un período tiempo determinado.

$$
S s p_{i \Delta}=\sum_{y=1}^{k}\left(A s_{i y t}-A s_{i y t-1}\right) p_{y}
$$

Donde:

As = Activo social

$y=$ Cada activo de la riqueza $\operatorname{social}(y=1 \ldots, k)$

$i \quad=$ Cada proceso $(i=1 \ldots, m)$

As ${ }_{y t} \quad=$ Activo social ( $y$ ) en el tiempo ( $t$ ) en el proceso (i)

$A s_{y t-1}=$ Activo social $(y)$ en el tiempo ( $\left.t-1\right)$ en el proceso (i)

$P_{y} \quad$ factor unidimensional de conversión de unidad de cada activo $(y)$ en la dimensión social de la riqueza en los procesos.

c. Medición de la Sustentabilidad Económica en un Proceso [Sep $\left.{ }_{\mathrm{i} \Delta}\right]$. Se calcula a través de la sumatoria de la sustentabilidad de cada activo de la riqueza económica en el proceso (i), multiplicado por su factor de conversión, medido en un período tiempo determinado.

$$
\operatorname{Sep}_{i \Delta}=\sum_{z=1}^{q}\left(A e_{i z t}-A e_{z t-1}\right) p_{z}
$$

Donde:

Ae = Activo económico

$z \quad=$ Cada recurso de la riqueza económica $(z=1 \ldots, q)$

$i \quad=$ Cada proceso $(i=1 \ldots, m)$

$A e_{z t}=$ Activo económico $(z)$ en el tiempo $(t)$ en el proceso ( $\left.i\right)$

$A z_{z t-1}=$ Activo económico (z) en el tiempo ( $\left.t-1\right)$ en el proceso (i)

$P_{z} \quad$ factor unidimensional de conversión de unidad de cada activo (z) en la dimensión económica de la riqueza en los procesos 
El esquema general de lo expuesto, se observa en la figura 1.

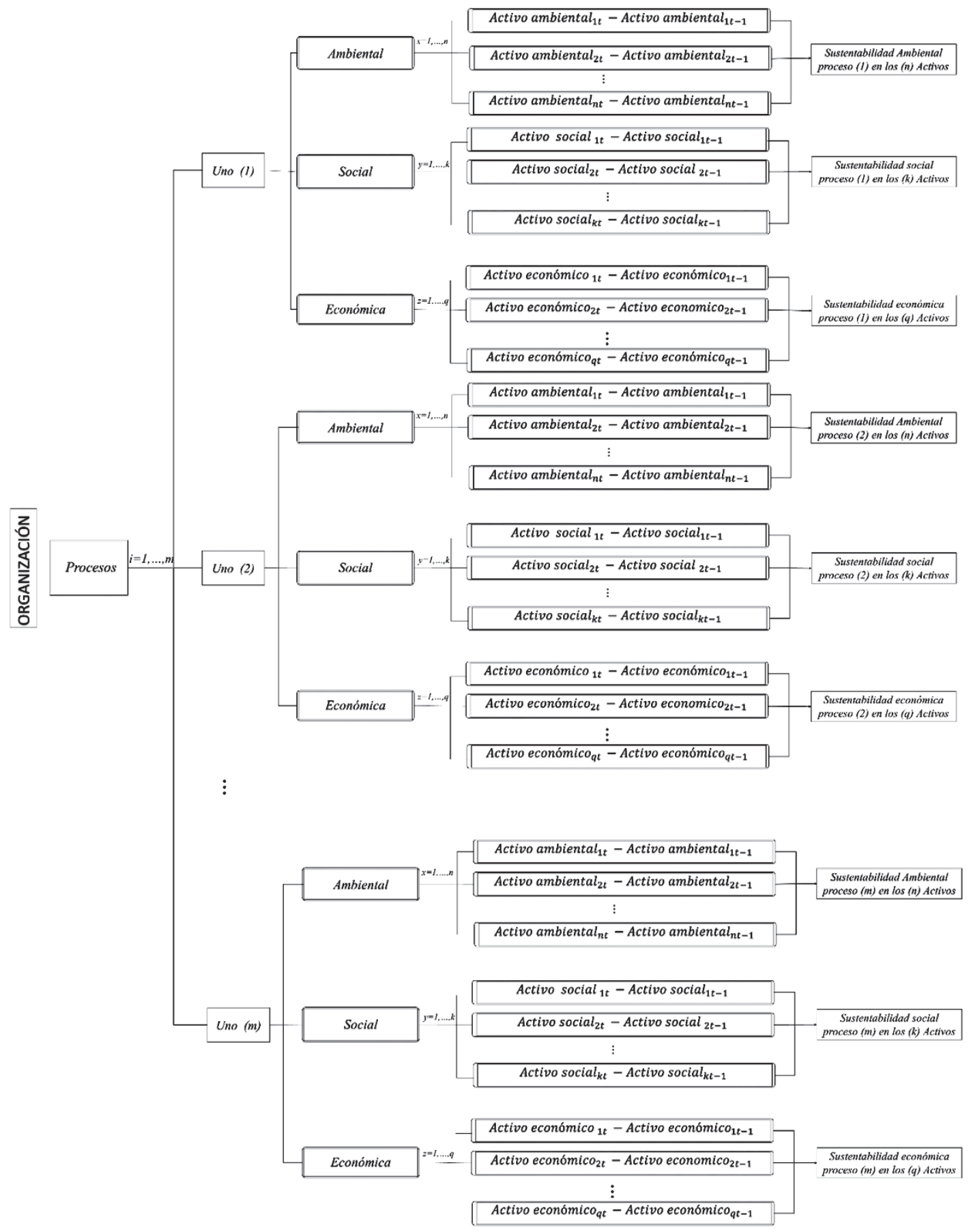

Figura 1. Medición de la sustentabilidad de la riqueza en los procesos Fuente: elaboración propia 


\section{Medición de la sustentabilidad de la riqueza en la organización a través de sus activos}

La medición de la sustentabilidad de la riqueza en la organización a través de los activos puede calcularse mediante dos metodologías:

a. Unidimensional de la riqueza.

b. Tridimensional en los procesos.

\section{Medición de la sustentabilidad unidimensional de la riqueza en la organización}

a. Medición de la sustentabilidad ambiental en la organización [ $\left.\mathrm{SaO}_{\Delta}\right]$ : Se calcula a través de la sumatoria de la sustentabilidad de cada activo de la riqueza ambiental en todos los procesos de la organización, medido en un período de tiempo determinado.

$$
\mathrm{Sao}_{\Delta}=\sum_{i=1}^{m} \sum_{x=1}^{n}\left(A a_{i x t}-A a_{i x t-1}\right) p_{x}
$$

Donde:

$$
\begin{aligned}
A a & =\text { Activo ambiental } \\
x & =\text { Cada activo de la riqueza en la dimensión ambiental }(x=1 \ldots, n) \\
i & =\text { Cada proceso }(i=1 \ldots, m) \\
A a_{x t}= & \text { Activo ambiental }(x) \text { en el tiempo }(t) \text { en el proceso }(i) \\
A a_{x t-1}= & \text { Activo ambiental }(x) \text { en el tiempo }(t-1) \text { en el proceso }(i) \\
P_{x} & =\text { factor unidimensional de conversión de unidad de cada activo }(x) \text { en la dimensión } \\
& \text { ambiental de la riqueza en los procesos }
\end{aligned}
$$

b. Medición de la sustentabilidad social en la organización [Sso $\left.{ }_{\triangle}\right]$ : Se calcula a través de la sumatoria de la sustentabilidad de cada activo de la riqueza social en todos los procesos de la organización, medido en un período de tiempo determinado.

$$
S s o_{\Delta}=\sum_{i=1}^{m} \sum_{y=1}^{k}\left(A s_{i y t}-A s_{i y t-1}\right) p_{y}
$$

Donde:

$$
\begin{array}{ll}
\text { As } & =\text { Activo social } \\
y & =\text { Cada activo de la riqueza social }(y=1 \ldots, k) \\
i & =\text { Cada proceso }(i=1 \ldots, m) \\
\text { As } & =\text { Activo social }(y) \text { en el tiempo }(t) \text { en el proceso }(i)
\end{array}
$$


$A s_{y t-1}=$ Activo social $(y)$ en el tiempo ( $\left.t-1\right)$ en el proceso (i)

$P_{y} \quad=$ factor unidimensional de conversión de unidad de cada activo $(y)$ en la dimensión social de la riqueza en los procesos.

c. Medición de la Sustentabilidad Económica en la Organización [Seo $]$ : Se calcula a través de la sumatoria de la sustentabilidad de cada activo de la riqueza económica en todos los procesos de la organización, medido en un período de tiempo determinado.

$$
\mathrm{Seo}_{\Delta}=\sum_{i=1}^{m} \sum_{z=1}^{q}\left(A e_{i z t}-A e_{i z t-1}\right) p_{z}
$$

Donde:

Ae = Activo económico

$z \quad=$ Cada recurso de la riqueza económica $(z=1 \ldots, q)$

$i \quad=$ Cada proceso $(i=1 \ldots, m)$

$A e_{z t}=$ Activo económico (z) en el tiempo ( $t$ ) en el proceso ( $\left.i\right)$

$A z_{z t-1}=$ Activo económico ( $z$ ) en el tiempo ( $\left.t-1\right)$ en el proceso (i)

$P_{z} \quad$ factor unidimensional de conversión de unidad de cada activo (z) en la dimensión económica de la riqueza en los procesos

\section{Medición de la sustentabilidad tridimensional en un proceso de la organización}

Medición de la sustentabilidad tridimensional en los procesos [Stpi $\Delta$ ]: Se calcula a través de la sumatoria de la sustentabilidad de la riqueza [fase activos] en las tres dimensiones en el proceso (i):

$$
\begin{aligned}
& S t p_{i \Delta}=\left(\left(\operatorname{Sap}_{i \Delta}\right) f_{x}\right) w_{x}+\left(\left(\operatorname{Ssp}_{i \Delta}\right) f_{y}\right) w_{y}+\left(\left(\operatorname{Sep}_{i \Delta}\right) f_{z}\right) w_{z} \\
& \operatorname{Stp}_{i \Delta}=\left(\left(\sum_{x=1}^{n}\left(A a_{i x t}-A a_{i x t-1}\right) p_{x}\right) f_{x}\right) w_{x}+\left(\left(\sum_{y=1}^{k}\left(A s_{i y t}-A s_{i y t-1}\right) p_{y}\right) f_{y}\right) w_{y} \\
& +\left(\left(\sum_{z=1}^{q}\left(A e_{i z t}-A e_{i z t-1}\right) p_{z}\right) f_{z}\right) w_{z}
\end{aligned}
$$

Donde:

$i=$ Cada proceso $(i=1 \ldots, m)$

$f_{x}=$ factor tridimensional de conversión de la riqueza ambiental

$f_{y}=$ factor tridimensional de conversión de la riqueza social 
$f_{z}=$ factor tridimensional de conversión de la riqueza económica

$w_{x}=$ factor de ponderación de la riqueza ambiental

$w_{y}=$ factor de ponderación de la riqueza social

$w_{z}=$ factor de ponderación de la riqueza económica

\section{Medición de la sustentabilidad tridimensional en la organización a través de sus activos}

La medición de la sustentabilidad tridimensional en la organización puede calcularse a través de dos metodologías, el resultado debe ser equivalente por las dos opciones:
a. Por dimensiones.
b. Por procesos.

Medición de la sustentabilidad tridimensional en la organización por dimensiones [Stods)

Se calcula a través de la sumatoria de la medición de la sustentabilidad en las tres dimensiones de la organización [fase activos]:

$$
\begin{gathered}
\operatorname{stod}_{\Delta}=\left(\left(\sum_{i=1}^{m} \sum_{x=1}^{n}\left(A a_{i x t}-A a_{i x t-1}\right) p_{x}\right) f_{x}\right) w_{x}+\left(\left(\sum_{i=1}^{m} \sum_{y=1}^{k}\left(A s_{i y t}-A s_{i y t-1}\right) p_{y}\right) f_{y}\right) w_{y} \\
+\left(\left(\sum_{i=1}^{m} \sum_{z=1}^{q}\left(A e_{i z t}-A e_{i z t-1}\right) p_{z}\right) f_{z}\right) w_{z}
\end{gathered}
$$

Donde

$f_{x}=$ factor tridimensional de conversión de la riqueza ambiental

$f_{y}=$ factor tridimensional de conversión de la riqueza social

$f_{z}=$ factor tridimensional de conversión de la riqueza económica

$w_{x}=$ factor de ponderación de la riqueza ambiental

$w_{y}=$ factor de ponderación de la riqueza social

$w_{z}=$ factor de ponderación de la riqueza económica 


\section{Medición de la sustentabilidad tridimensional en la organización por procesos}

Se calcula a través de la sumatoria de la medición de la sustentabilidad tridimensional de la riqueza en todos los procesos.

$$
\begin{gathered}
\operatorname{Stop}_{\Delta}=\sum_{i=1}^{m}\left[\left(\left(\sum_{x=1}^{n}\left(A a_{i x t}-A a_{i x t-1}\right) p_{x}\right) f_{x}\right) w_{x}+\left(\left(\sum_{y=1}^{k}\left(A s_{i y t}-A s_{i y t-1}\right) p_{y}\right) f_{y}\right) w_{y}\right. \\
\left.,+\left(\left(\sum_{z=1}^{q}\left(A e_{i z t}-A e_{i z t-1}\right) p_{z}\right) f_{z}\right) w_{z}\right]
\end{gathered}
$$

La medición contable de la sustentabilidad organizacional [fase activos] corresponde a la primera fase de la medición de la sustentabilidad tridimensional de la organización, la segunda fase corresponde al método de circulación [fase ingresos y egresos] tal como se ilustra en la tabla 2.

Notas aclaratorias:

- La fase 1 [activos] utiliza el término activos, en sentido similar, la fase 2 [ingresos-egresos] utiliza el término recurso.

- La utilización de la letra griega $[\Delta]$ en la fase 1 diferencia las mediciones de sustentabilidad con respecto a la fase 2 ; en la primera se calcula a través de la diferencia que existe entre las mediciones de un activo en dos fechas específicas de tiempo. La segunda obtiene la medición de la sustentabilidad como resultado de la diferencia entre los ingresos y los egresos de los recursos en un período de tiempo determinado. 
Tabla 2. Medición contable de la sustentabilidad organizacional, fase activos

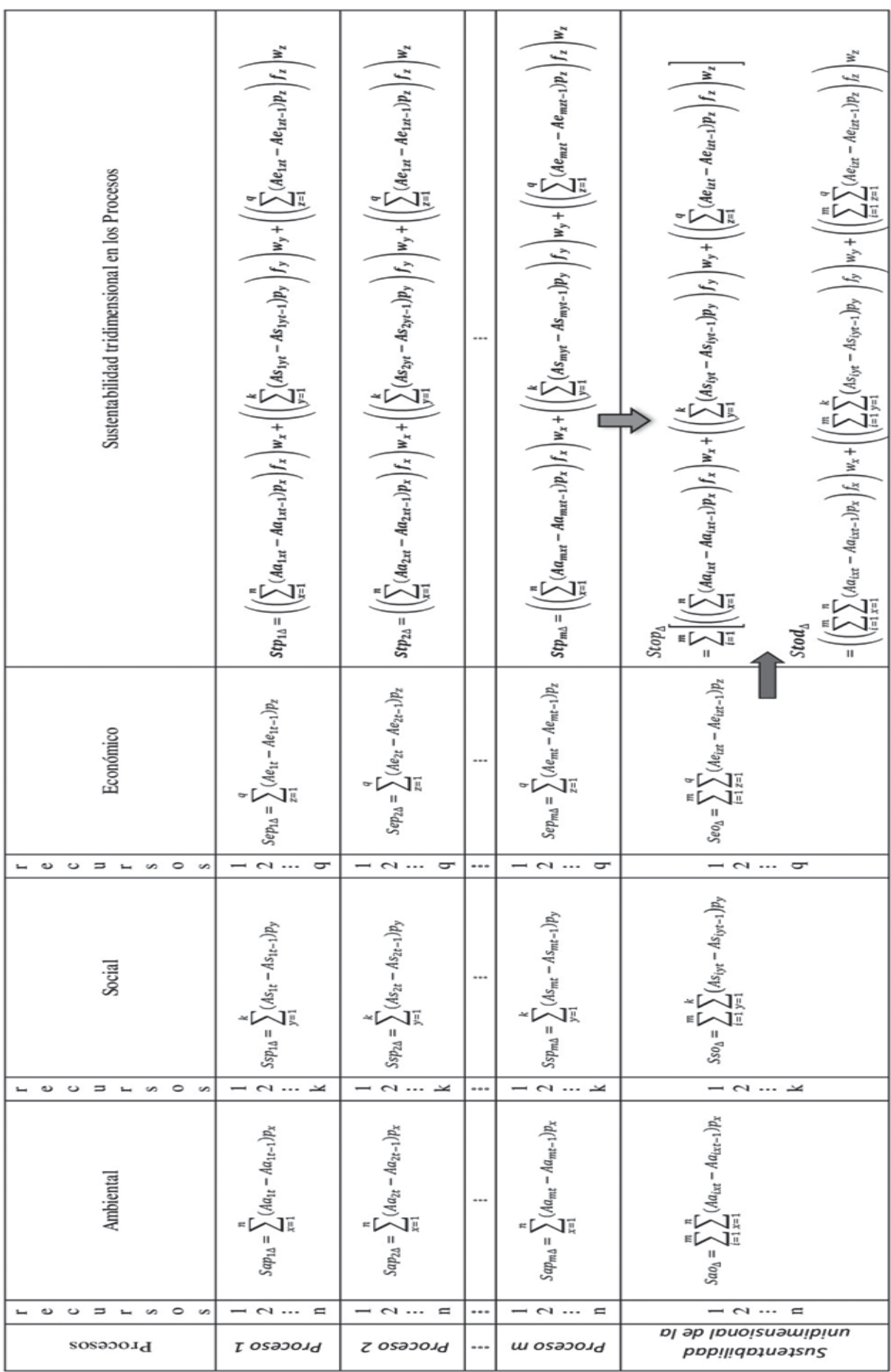

Fuente: elaboración propia 
Tabla 3. Medición contable de la sustentabilidad organizacional [fase ingresos y egresos]

\begin{tabular}{|c|c|c|c|c|c|}
\hline 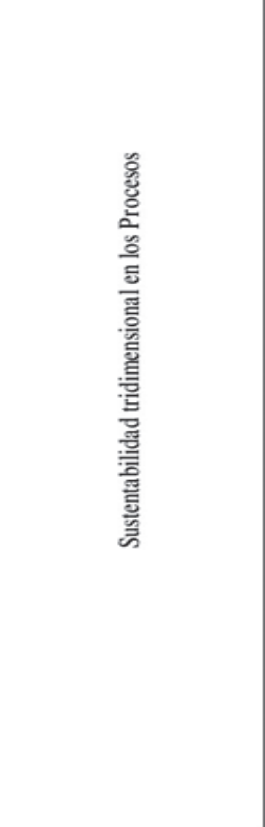 & 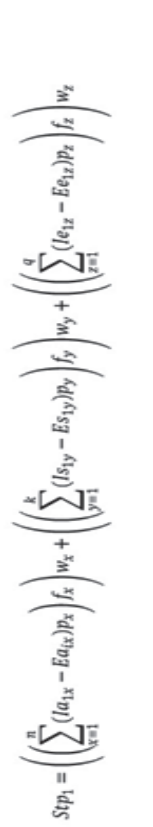 & 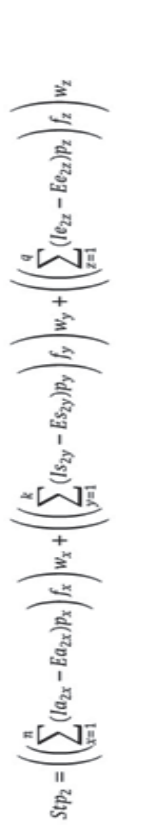 & $\ldots$ & 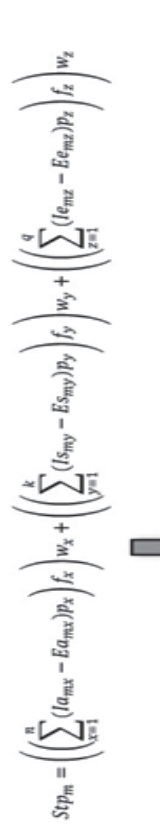 & 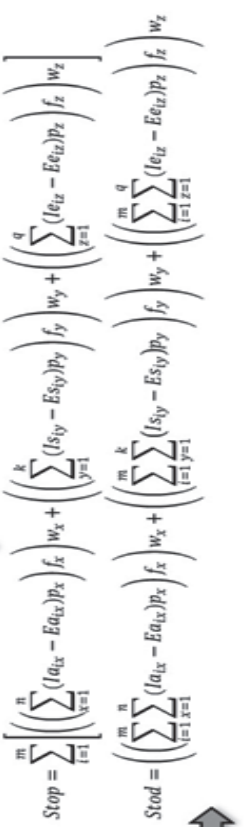 \\
\hline $\begin{array}{l}\text {. } \\
\text { 㤩 } \\
\text { 总 }\end{array}$ & 竎 & 产 & ... & 望 & 势 \\
\hline$-00=-\infty 00$ & $-N \cdots \sigma$ & $-4 \cdots \sigma$ & $\cdots$ & $-N \ldots \sigma$ & $-4 \cdots \sigma$ \\
\hline 跘 & 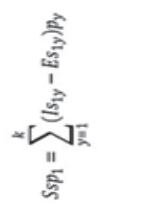 & 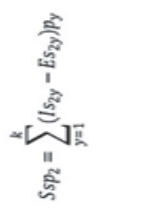 & $\ldots$ & 章 & 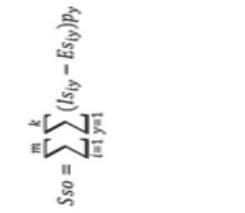 \\
\hline $200=-n 0 n$ & $-n \cdots \Psi$ & $-N \cdots \ddot{x}$ & $\ldots$ & $-N \cdots \ddot{x}$ & $-n \cdots x$ \\
\hline 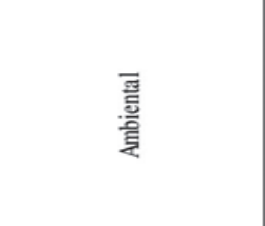 & 童 & " & $\cdots$ & = & 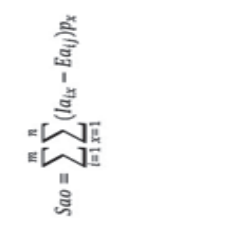 \\
\hline L0 $0=-\infty 0 n$ & $-N \cdots=$ & $-N \cdots=$ & $\ldots$ & $-N \cdots=$ & $-n \ldots=$ \\
\hline sosəoos $_{d}$ & I osøoosd & z osəoosd & $\cdots$ & u osəoo. $x_{d}$ & 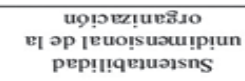 \\
\hline
\end{tabular}

Fuente: elaboración propia 
Las nomenclaturas usadas en la tabla 3 son las siguientes:

Dimensión Ambiental (Biocontabilidad)

$X=$ Cada recurso de la riqueza en la dimensión ambiental $(x=1 \ldots, n)$

$S a_{x}=$ Sustentabilidad del recurso $(x)$ en la dimensión ambiental

$I a_{x}=$ Ingreso del recurso $(x)$ en la dimensión ambiental

$E a_{x}=$ Egreso del recurso $(x)$ en la dimensión ambiental

$S_{a p}{ }_{i}=$ Medición de la sustentabilidad ambiental en un Proceso

Dimensión social (Sociocontabilidad)

$y=$ Cada recurso de la riqueza en la dimensión social $(y=1 \ldots, k)$

$S_{s_{y}}=$ Sustentabilidad del recurso $(y)$ en la dimensión social

Is $s_{y}=$ Ingreso del recurso $(y)$ en la dimensión social

$E s_{y}=$ Egreso del recurso (y) en la dimensión social

$S_{s p_{i}}=$ Medición de la sustentabilidad social en un Proceso

Dimensión económica (Contabilidad económica)

$z \quad=$ Cada recurso de la riqueza en la dimensión económica $(z=1 \ldots, q)$

$\mathrm{Se}_{z}=$ Sustentabilidad del recurso (z) en la dimensión económica

$\mathrm{Ie}_{z}=$ Ingreso del recurso (z) en la dimensión económica

$E e_{z}=$ egreso del recurso (z) en la dimensión económica

Sep $_{i}=$ Medición de la Sustentabilidad económica en un Proceso

\section{Resultados de la medición de la sustentabilidad unidimensional y tridimensional de la riqueza en la organización}

\section{Resultado de la medición de la sustentabilidad unidimensional en la organización}

El resultado de la medición de la sustentabilidad de los activos en cada dimensión dimensional en la organización presenta tres opciones:

- Sustentabilidad [+]: el resultado de la medición de la sustentabilidad de la riqueza unidimensional [fase activos] de valor positivo, indica que se ha presentado un incremento cualitativo y/o cuantitativo de la riqueza en el tiempo t con respecto a $\mathrm{t}-1$.

- Estabilidad [0]: el resultado de la medición de la sustentabilidad de la riqueza unidimensional [fase activos] con valor cero, indica que la riqueza conserva un mantenimiento dinámico cualitativo y/o cuantitativo, conservando un equilibrio en t con respecto a $\mathrm{t}-1$. 
- Insustentabilidad [-]: el resultado de la medición de la sustentabilidad de la riqueza unidimensional [fase activos] con valor negativo, indica que se ha presentado un decremento cualitativo y/o cuantitativo de la riqueza en el tiempo t con respecto a $\mathrm{t}-1$. (Ver tabla 4).

Tabla 4. Posibilidades de calificación de la sustentabilidad de la riqueza unidimensional y tridimensional en la organización

\begin{tabular}{|c|c|c|c|c|c|c|c|c|c|c|c|c|c|c|c|c|c|c|c|c|c|c|c|c|c|c|c|}
\hline & 1 & 2 & 3 & 4 & 5 & 6 & 7 & 8 & 9 & 10 & 11 & 12 & 13 & 14 & 15 & 16 & 17 & 18 & 19 & 20 & 21 & 22 & 23 & 24 & 252 & 262 & 27 \\
\hline Ambiental & + & + & + & + & + & + & + & + & + & 0 & 0 & 0 & 0 & 0 & 0 & 0 & 0 & 0 & - & - & - & - & - & - & - & - & - \\
\hline Social & + & + & + & 0 & 0 & 0 & - & - & - & + & + & + & 0 & 0 & 0 & - & - & - & + & + & + & 0 & 0 & 0 & - & - & - \\
\hline Económico & + & 0 & - & 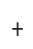 & 0 & - & + & 0 & - & + & 0 & - & + & 0 & - & + & 0 & - & + & 0 & - & + & 0 & - & +0 & 0 & - \\
\hline
\end{tabular}

Fuente: elaboración propia

\section{Resultado del desempeño organizacional en la sustentabilidad tridimensional}

El cálculo de la medición de la sustentabilidad de la riqueza tridimensional en la organización, se obtiene de la sumatoria ponderada de las mediciones de la sustentabilidad unidimensional en la organización o por la sumatoria de la sustentabilidad tridimensional de los procesos. La pirámide de la sustentabilidad establece que el nivel de importancia de las dimensiones de la riqueza en su orden es ambiental, social y económica [criterio axiológico].

Los resultados posibles de medición de la sustentabilidad organizacional son los siguientes:

a. Sustentabilidad tridimensional

1. Sustentabilidad tridimensional positiva $(+++)$ [1 opción].

b. Sustentabilidad parcial

2. Sustentabilidad bidimensional con estabilidad unidimensional [3 opción].

3. Sustentabilidad bidimensional con insustentabilidad unidimensional [3 opción].

4. Estabilidad bidimensional con sustentabilidad unidimensional [3 opción].

5. Estabilidad bidimensional con insustentabilidad unidimensional [3 opción].

6. Insustentabilidad bidimensional con estabilidad unidimensional [3 opción].

7. Insustentabilidad bidimensional con sustentabilidad unidimensional [3 opción].

8. Sustentabilidad unidimensional con estabilidad unidimensional e insustentabilidad unidimensional [6 opciones].

c. Estabilidad tridimensional

9. Estabilidad en las tres dimensiones (000) [1 opción].

d. Insustentabilidad tridimensional

10. Insustentabilidad en las tres dimensiones (- - ) [1 opción]. 


\section{Conclusiones}

- El resultado de la evaluación de la gestión de la riqueza por parte de la organización a través de la medición contable, contribuye a la toma de decisiones de los grupos de interés, en virtud de las prioridades y necesidades de estos. La orientación ética del sistema social debe priorizar las organizaciones socio-ambientalmente responsables, asimismo, deberá sancionar aquellas que adelantan prácticas que destruyen la riqueza en sus diferentes manifestaciones.

- Algunos activos que conforman la riqueza ambiental también son considerados como activos en la riqueza social y económica; corresponde al sujeto decisor moral definir la disposición-uso del mismo, en el marco de opciones múltiples complementarios y/o excluyentes. La riqueza tiene un carácter integral, la división en dimensiones permite una evaluación en detalle de los impactos organizacionales, pero es una abstracción de una realidad que actúa de forma holística, indivisible e interrelacionada.

- La protección, cuidado y mantenimiento dinámico de la riqueza requiere la medición de la existencia, circulación y comportamiento de los mismos en el tiempo; si estos no son medidos y sus cambios no son contabilizados, existirán limitantes para la implementación de acciones tendientes a su sustentabilidad. La contabilidad es un punto necesario, pero no suficiente para la sustentabilidad.

- Las unidades de medida de los activos y de las dimensiones de la riqueza son múltiples, surgiendo dos opciones para el análisis, la primera, la utilización de una unidad única que permita expresar todas las manifestaciones de riqueza en criterios estándar; la segunda opción, corresponde a la utilización de metodologías de análisis que incluyan las diversas unidades de medida que han permitido la representación de las riquezas objeto de estudio.

- La medición de la sustentabilidad de la organización debe de ser integral, la presentación de información en una única dimensión es parcial, reduccionista y no refleja el comportamiento sustentable de la organización. La evaluación de la gestión organizacional en el control de la riqueza exige la lectura conjunta de las mediciones de la sustentabilidad en las tres dimensiones.

- El cálculo de la sustentabilidad de la organización obtenido a través de los resultados unidimensionales y la división de la actividad organizacional en procesos, permite identificar en cada proceso y en cada dimensión el impacto en términos de sustentabilidad, estabilidad o insustentabilidad de la riqueza.

- La T3C fundamenta que la contabilidad es una ciencia moral, de carácter normativo, orientada a un deber ser, de búsqueda de mejoramiento permanente de las condiciones de vida en el planeta; reclama una visión biocéntrica que reconoce el derecho igualitario a existir que deben tener todas las especies, se aleja de visiones antropocéntricas que consideran al hombre como centro del universo o consideran la especie humana como la de mayor importancia. La sustentabilidad es concebida como un bien en sí mismo, independiente de los beneficios que represente para el hombre la conservación, protección y mantenimiento dinámico de la riqueza en sus dimensiones ambiental, social y económica. 
- La contabilidad evalúa el desempeño organizacional en los campos ambiental, social y económico, los resultados obtenidos cumplen dos funciones, la primera de rendición de cuentas por parte de la organización ante la sociedad; la segunda permitirá a partir de los resultados integrales de la contabilidad, que los diferentes agentes sociales puedan tomar decisiones con suficiente información. El quehacer organizacional será evaluado moral, social, política y económicamente, los estados financieros tradicionales han realizado evaluaciones parciales (únicamente financieras), situación que llevó a que la rendición de cuentas fuera parcial y la toma de decisiones se hiciera con información incompleta.

- El tránsito de la contabilidad financiera como sistema contable de implementación generalizada, hacia la contabilidad para la sustentabilidad ambiental y social, implica la reconstrucción plena del saber contable. La teoría general contable (TGC) deberá ser repensada y reformulada, de forma que permita la inclusión de las revisiones ontológicas, epistemológicas, teleológicas y axiológicas del saber contable. Los marcos conceptuales de la contabilidad, como parte de los sistemas aplicados, deberán ser ajustados conforme a los criterios de la TGC. El campo técnico-procedimental se desarrollará de conformidad y siguiendo en línea deductiva los criterios establecidos en la TGC y los marcos conceptuales, dependiendo el campo de implementación específica.

- La contabilidad, desde la visión de la T3C en orden de prioridad, propende por la sustentabilidad de la riqueza ambiental, social y económica en condición de recursos controlados por las organizaciones; se considera que dicha sustentabilidad contribuye a la sostenibilidad organizacional (permanencia con óptimo desempeńo) y tal situación llevará a una economía local, nacional e incluso global, satisfactoria en términos de construcción colectiva de condiciones propicias para la prosperidad y la vida en todas sus manifestaciones [concepto crítico-oposición al desarrollo sostenible].

\section{Referencias}

1. Agencia Europea de Medio Ambiente. (2013). Un marco experimental para la contabilidad y el capital ecosistémico en Europa. Madrid: Ministerio de Agricultura, Alimentación y Medio Ambiente.

2. Biondi Mario. (2008). Bases teóricas del capital computable para medir los resultados de un periodo. Uruguay: Universidad de Concepción del Uruguay.

3. Boada Alejandro; Rocchi Simona y Kuhndt Michel. (2005). Negocios y sostenibilidad más allá de la gestión ambiental. Bogotá: PGC.

4. Brundtland, Gro Harlem. (1987). Nuestro Futuro Comín. Oslo: UN.

5. Cańibano Calvo, Leandro. (2000, abril-junio). El medio ambiente en la contabilidad de las empresas. En: Revista Legis del Contador 2, 81-96.
6. Caro, Juan Carlos. (2011). Contabilidad Ambiental. Ventajas del desarrollo sustentable. Buenos Aires: Edicon.

7. Fowler Newton, Enrique. (2007). Cuestiones contables fundamentales. Buenos Aires: La Ley.

8. Gallopín, Gilberto. (2003). Sostenibilidad y desarrollo sostenible: un enfoque sistémico. Santiago de Chile: CEPAL.

9. García Benau, María Antonia. (2004). Introducción a la contabilidad fnnanciera: un enfoque internacional. Barcelona: Ariel.

10. García Casella, Carlos Luis. (2001). Elementos para una teoría general de la contabilidad. Buenos Aires: La Ley.

11. García García, Moisés. (1997). Ensayos sobre teoría de la contabilidad, origen, desarrollo y contenido actual del análisis circulatorio. Madrid: ICAC.

12. Geba Norma, Fernández Liliana y Sebastián Mónica. (2008, julio-septiembre). El proceso contable en la especia- 
lidad socio-ambiental. En: Revista de Legis de Contabilidady Auditoría 35, 117-152.

13. International Integrated Reporting Council (IIRC). (2013). The international Framework. IIRC.

14. IFRSF (2014). Normas Internacionales de Información Financiera. London: IFRS.

15. Labandeira Xavier; León Carmelo y Vázquez María. (2006). Economía ambiental. Madrid: Pearson.

16. López de Sá, Antonio. (1992). Fundamentos lógicos de la teoría de las funciones sistemáticas del patrimonio hacendal. Cuadernos, 23, 13-45.

17. López de Sá, Antonio. (1995). Autonomía y calidad científica de la contabilidad. Revista de la Facultad de Contaduría Pública UNAB.

18. Mattessich Richard (2002). Contabilidad y métodos analiticos. Buenos Aires: La Ley.

19. Mejía Soto, Eutimio (2010). Contabilidad ambiental. Armenia: FIDESC.

20. Mejía Soto, Eutimio; Montes Salazar, Carlos Alberto; Montilla Galvis, Omar de Jesús. (2010). Análisis de los métodos de medición de las cuentas ambientales en el modelo contable financiero y concepciones alternativas. En: Revista Entramado 2, 106-128.

21. Mejía Soto, Eutimio; Montes Salazar, Carlos Alberto y Botero Echeverry, David. (2010). Programas de Investigación en Contabilidad. Cali. Artes Gráficas.

22. Mejía Soto, Eutimio (2011). Análisis de los criterios de reconocimiento de las cuentas ambientales en los estándares internacionales de contabilidad y reportes financieros IASIFRS. Universidad de Manizales: Lúmina, 114-137.

23. Mejía Soto, Eutimio y Montes Salazar, Carlos Alberto (2011). Estructura general de la teoría pura, los modelos y sistemas en contabilidad. Encuentro de la Red de Filosofía, Teoría y Educación en Administración. Universidad Nacional de Colombia. 11, 12 y 13 de mayo, Manizales.

24. Mejía Soto, Eutimio y Montes Salazar, Carlos Alberto (2011a). Perú. Fundamentación teórica de los modelos en contabilidad. En: Revista Cientifica Estudiantil Innovación Contable 1,7-18.

25. Mejía Soto, Eutimio y Montes Salazar, Carlos Alberto. (2012, enero-marzo). Análisis de los conceptos de capital y mantenimiento y su relación con la riqueza ambiental, Revista Internacional Legis de Contabilidad y Auditoría, 49, $115-154$
26. Mejía Soto, Eutimio; Montes Salazar, Carlos Alberto y Arango Mediana, Deyci (2012). Aproximación al sistema de contabilidad ambiental y económica integrada SCAEI de la ONU. Sinapsis. EAM, 18, 30 .

27. Mejía Soto, Eutimio y Vargas Marín, Luis Alberto (2012, enero-diciembre). Contabilidad para la sostenibilidad ambiental y social. Revista Lúmina, 13, 48-70.

28. Mejía Soto, Eutimio; Montes Salazar, Carlos Alberto y Mora Roa, Gustavo. (2013). Estructura conceptual de la teoría tridimensional de la contabilidad. En: Contexto, 2, 25-44.

29. Mejía Soto, Eutimio; Mora Roa, Gustavo y Montes Salazar, Carlos Alberto. (2013). Adscripción de la contabilidad en la estructura general del conocimiento. En: Revista Cuadernos de Contabilidad, 34, 159-187.

30. Mejía Soto, Eutimio. (2014, enero-diciembre). Biocontabilidad: hacia una definición de una nueva disciplina contable. Revista Lúmina, 15, 106-129.

31. Mejía Soto, Eutimio (2014). La Teoría Tridimensional de la Contabilidad: apuntes desde la economía ecológica. Martínez y Roca, 27, 181-208.

32. Mejía Soto, Eutimio; Montilla Galvis, Omar; Montes Salazar, Carlos Alberto y Mora Roa, Gustavo. (2014). Teoría tridimensional de la contabilidad T3C (versión 2.0): desarrollos, avances y temas propuestos. Revista Libre Empresa, 22, 95-120.

33. Mejía Soto, Eutimio; Montilla Galvis, Omar; Montes Salazar, Carlos Alberto y Mora Roa, Gustavo. (2014a). Teoría tridimensional de la contabilidad. Pereira. Universidad Libre de Colombia.

34. Mejía Soto, Eutimio; Mora Roa, Gustavo, Montes Salazar, Carlos Alberto y Montilla Galvis, Omar. (2014). Marco conceptual de la biocontabilidad. Sinapsis 6, 147-178.

35. Mejía Soto, Eutimio. (2015). Aportes a la biocontabilidad desde la bioeconomía de Georgescu-Roegen. Revista Asuntos, 28, 169-191.

36. Mejía Soto, Eutimio y Serna Mendoza, Ciro. (2015). La contabilidad en función de la sustentabilidad: una mirada desde el desarrollo económico alternativo. Quipukamayoc, 23, (44), 109-118.

37. Mejía Soto, Eutimio; Montes Salazar, Carlos Alberto y Mora Roa, Gustavo. (2015). Marco conceptual de la contabilidad ambiental. Armenia: Universidad del Quindío

38. Mejía Soto, Eutimio; Montilla Galvis, Omar; Montes Salazar, Carlos Alberto y Mora Roa, Gustavo. (2015) Teoría 
tridimensional de la contabilidad. Versión 2.0. Pereira. Universidad Libre de Colombia.

39. Mejía Soto, Eutimio y Quintero Arenas, Kimberly. (2015). Biocontabilidad: un enfoque para la sustentabilidad. En: Muńoz Velasco, Alfredo. Economía, medio ambiente y desarrollo sostenible. Un mapa de la cuestión. Neiva. Universidad Surcolombiana. Memorias del I Simposio Internacional sobre Gestión del Conocimiento, Economía y Medio Ambiente para el Desarrollo Sostenible, septiembre 3 y 4 de 2015.

40. Montes Salazar, Carlos Alberto; Mejía Soto, Eutimio y Montilla Galvis, Omar de Jesús. (2005). Contabilidad emergente. Cali: Universidad Libre.

41. Montes Salazar, Carlos Alberto; Mejía Soto, Eutimio y Valencia Salazar, Jhon Jairo. (2006) Paradigmas en contabilidad. Cali: Artes Gráficas del Valle.
42. Mora Roa, Gustavo; Montes Salazar, Carlos Alberto y Mejía Soto, Eutimio. (2005). Efectos de la regulación contable internacional. Armenia: Universidad del Quindío.

43. Nortth, Doguglass. (2012). Instituciones, cambio institucional y desempeño económico. México. FCE.

44. Ostengo Héctor. (2007). El sistema de información contable. Buenos Aires: Osmar Buyatti.

45. Ostrom, Elionor. (2011). El Gobierno de los bienes comunes. Evolución de las instituciones de acción colectiva. México, 2011.

46. Schuschny, Andrés y Soto, Humberto. (2009). Guia metodológica de indicadores compuestos de desarrollo sostenible. Santiago de Chile: CEPAL.

47. Wirth, María Cristina. (2001). Acerca de la contabilidad en el campo del conocimiento. Buenos Aires: La Ley. 